\title{
1 Profiling the initial burst of beneficial genetic diversity 2 in clonal cell populations to anticipate evolution
}

4 Daniel E. Deatherage and Jeffrey E. Barrick*

6 Department of Molecular Biosciences, Center for Systems and Synthetic Biology,

7 The University of Texas at Austin, Austin, Texas 78712, U.S.A.

8

$9 \quad *$ Email: jbarrick@,cm.utexas.edu 
10 Data Availability Statement: DNA sequence files are available from the NCBI Sequence Read

11 Archive (accession number PRJNA601748). Code and processed data files are available on

12 GitHub (https://github.com/barricklab/adaptome-capture).

13

14 Funding: This work was supported by the Cancer Prevention \& Research Institute of Texas

15 (CPRIT) (RP130124), the National Institutes of Health (R00-GM087550), the National Science

16 Foundation (CBET-1554179 and DEB-1813069), and the NSF BEACON Center for the Study of

17 Evolution in Action (DBI-0939454). D.E.D. acknowledges support from a University of Texas at

18 Austin CPRIT research traineeship (RP101501). The funders had no role in study design, data

19 collection and analysis, decision to publish, or preparation of the manuscript.

20

21 Competing interests: J.E.B. is the owner of Evolvomics LLC. D.E.D. has been a paid

22 consultant for Evolvomics LLC. 


\section{Abstract}

25 Clonal populations of cells continuously evolve new genetic diversity, but it takes a significant

26 amount of time for the progeny of a single cell with a new beneficial mutation to outstrip both its

27 ancestor and competitors to fully dominate a population. If these driver mutations can be

28 discovered earlier — while they are still extremely rare — and profiled in large numbers, it may be

29 possible to anticipate the future evolution of similar cell populations. For example, one could

30 diagnose the likely course of incipient diseases, such as cancer and bacterial infections, and

31 better judge which treatments will be effective, by tracking rare drug-resistant variants. To test

32 this approach, we replayed the first 500 generations of a >70,000-generation Escherichia coli

33 experiment and examined the trajectories of new mutations in eight genes known to be under

34 positive selection in this environment in six populations. By employing a deep sequencing

35 procedure using unique molecular identifiers and target enrichment we were able to track 301

36 beneficial mutations at frequencies as low as $0.01 \%$ and infer the fitness effects of 240 of these.

37 Distinct molecular signatures of selection on protein structure and function were evident for the

38 three genes in which beneficial mutations were most common ( $\operatorname{nadR}, p y k F$, and topA). We

39 detected mutations hundreds of generations before they became dominant and tracked beneficial

40 alleles in genes that were not mutated in the long-term experiment until thousands of generations

41 had passed. This type of targeted adaptome sequencing approach could function as an early

42 warning system to inform interventions that aim to prevent undesirable evolution. 


\section{Introduction}

44 New genetic variation naturally arises in lineages of cells and organisms during genome

45 replication and repair. These de novo mutations are the main drivers of adaptive evolution in

46 many populations, particularly those with little or no recombination or standing genetic variation.

47 In large laboratory populations of asexual microbes, numerous lineages with different beneficial

48 mutations arise and contend within a population before any one outcompetes the ancestor and its competitors (Good et al., 2017; Lang et al., 2013; Maddamsetti et al., 2015). This 'clonal

interference' leads to heterogeneous populations with many lineages simultaneously adapting via

51 distinct sets of mutations, though often these mutations are in a small subset of genes that are

52 under the strongest selection (Desai et al., 2012; Gerrish and Lenski, 1998; Park and Krug,

53 2007).

In human cancers and chronic microbial infections, single cells clonally expand in a similar solid tumors and blood cancers have been shown to be genetically heterogeneous (Marusyk et

61 Pseudomonas aeruginosa and other bacteria that persistently infect the lungs of cystic fibrosis

62 patients become increasingly invasive and antibiotic resistant over time (Marvig et al., 2015;

63 Stefani et al., 2017; Winstanley et al., 2016). In these cases, there are also specific genetic loci

64 that are repeatedly mutated in different individuals. Better predicting the future evolution of each 
65 of these types of cell populations and others would inform treatment decisions and improve

66 medical outcomes.

67 Cells used in biomanufacturing are also prone to evolving unwanted genetic heterogeneity

68 (Renda et al., 2014; Rugbjerg and Sommer, 2019). Typically, these cells have been heavily

69 engineered to optimize the titer of a product of interest at the expense of rapid cellular replication

70 (Lee and Kim, 2015; Nielsen and Keasling, 2016). Therefore, there are strong selective pressures

71 for 'escape mutations' that cause production to decline. Usually escape mutations directly

72 inactivate one or more key genes in the engineered pathway. The resulting nonproducing cells

73 can become dominant during the many cell divisions that are necessary to scale these processes

74 up to large bioreactors (Rugbjerg et al., 2018; Sandoval et al., 2014; Zelder and Hauer, 2000).

75 The ability to predict the future evolution of nonproducing cells before attempting scale-up could

76 guide strain design decisions and thereby improve the efficiency of industrial processes.

77 Evolution experiments conducted in laboratory environments reproduce key aspects of

78 microbial evolution that are observed in chronic infections and bioreactors (Barrick and Lenski,

79 2013; Gresham and Dunham, 2014). Certain aspects of genomic and phenotypic evolution in

80 these systems are surprisingly predictable while others are not (Barrick, 2020; Cvijović et al.,

81 2018; Furusawa et al., 2018; McDonald, 2019; Rainey et al., 2017). In theory, profiling many

82 rare mutations in the earliest stages of clonal interference using high-throughput DNA

83 sequencing should allow one to anticipate the future evolution of a population and other

84 populations that evolve in similar environments. Existing studies have generally been limited to

85 reliably identifying mutations that are present in at least one sample at a frequency above $\sim 1$ -

$8610 \%$ (due to sequencing depth costs and per-base error rates) when they have already succeeded

87 in becoming dominant (Barrick and Lenski, 2009; Chubiz et al., 2012; Good et al., 2017; Lang et 
al., 2013; Traverse et al., 2013). Theory and simulations predict that many more highly

beneficial mutations evolve in these populations but never reach such high frequencies before they are driven extinct (Desai et al., 2012; Gerrish and Lenski, 1998), and recent studies that track the evolution of barcoded lineages of microbes show that this is the case (Levy et al., 2015; Venkataram et al., 2016). sets of beneficial mutations found in three of these genes made it possible to understand the

100 long history of a $>70,000$-generation $E$. coli evolution experiment that used the same ancestral

101 strains and nearly identical culture conditions (Lenski et al., 1991), we are able to evaluate the

102 potential of this type of targeted adaptome analysis for anticipating the evolution of cell 103 populations.

\section{Results}

\section{Replaying the beginning of a long-term evolution experiment}

107 We initially examined the evolution of nine replicate E. coli populations that were propagated

108 via daily serial transfers in glucose-limited minimal medium for 500 generations. Our

109 experiment used the same E. coli strains as the Lenski long-term evolution experiment (LTEE)

110 and similar growth conditions (see Methods). Each population was inoculated with a 50/50 
111 mixture of the two neutrally marked LTEE ancestor strains to visualize the initial selective sweep

112 (Hegreness et al., 2006). Most populations maintained a roughly equal representation of

113 descendants of both ancestral strains through the first 300 generations (Fig. 1). These dynamics

114 are in agreement with what has previously been observed in studies of the LTEE, where few

115 mutations reach a high frequency in the first few hundred generations of evolution (Good et al.,

116 2017). We did not further analyze three populations. Two of these (A4 and A5) were

117 purposefully omitted because they exhibited early sweeps of one marker type, which indicates

118 that their dynamics might have been dominated by one or a few "jackpot" mutations that

119 occurred very early during outgrowth of these populations from single cells. The third omitted

120 population (A8) exhibited typical marker dynamics.

121
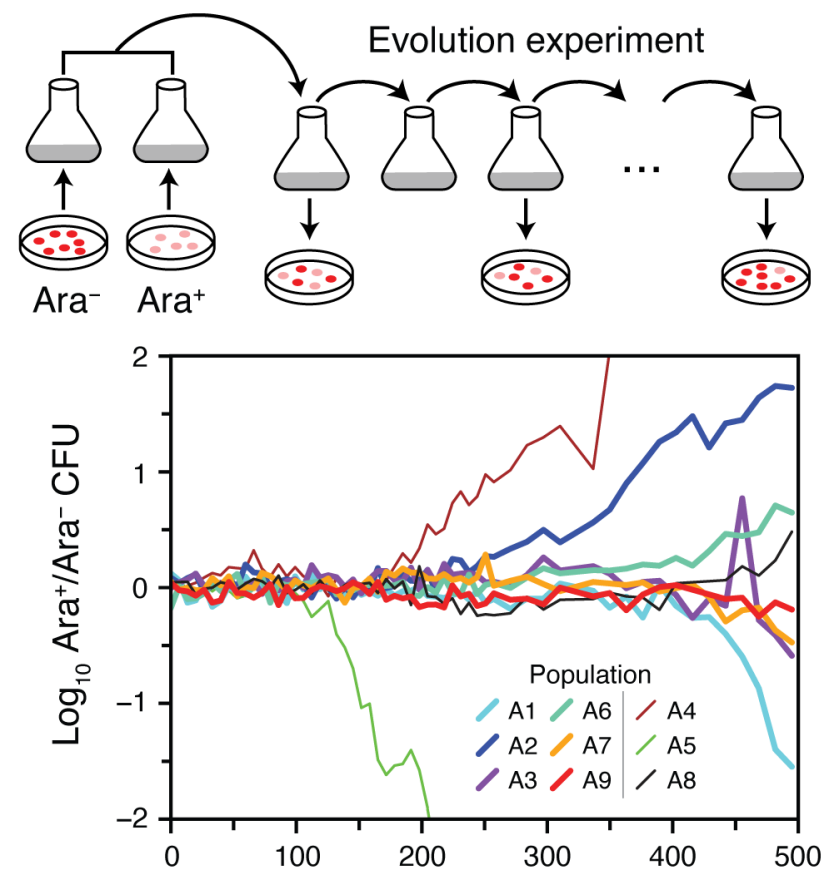

Generations of evolution

123 Figure 1. Replaying the first selective sweep of a long-term evolution experiment. Nine $E$. coli populations were initiated from equal mixtures of two variants of the ancestral strain that differ in a neutral genetic marker for arabinose utilization (Ara). We observed the evolutionary 
127 transfers by periodically plating dilutions of each population on indicator agar. The ratio of Ara ${ }^{+}$

128 cells (pink colonies) to Ara ${ }^{-}$cells (red colonies) diverges from 1:1 when descendants of one ancestor type accumulate enough of a fitness advantage due to de novo beneficial mutations that they take over. We focused further analysis on six of the nine populations (thick lines).

\section{Reconstructing the trajectories of new beneficial mutations}

133 We next performed deep sequencing of eight genes at $\sim 25$ generation increments over the entire

134500 generations of the evolution experiment for four of the six populations that we examined

135 further. These eight genes (nadR, pykF, topA, spoT, fabR, ybaL, hslU, and iclR) are known to be

136 targets of selection in the LTEE (Good et al., 2017; Tenaillon et al., 2016). Illumina libraries

137 containing unique molecular identifiers (Schmitt et al., 2012) were prepared for sequencing and

138 enriched for the regions of interest using solution based hybridization (Bainbridge et al., 2010).

139 Consensus sequence reads were generated based on groups of reads with identical unique

140 molecular identifiers and aligned to the $E$. coli genome to predict mutations, including using

141 split-read mapping to identify transposon insertions and large deletions (Fig. 2A). The

142 enrichment procedure was effective: an average of $73.5 \%$ of consensus reads per sample mapped

143 to the targeted regions that together constitute only $0.780 \%$ of the $4.63 \mathrm{Mbp}$ genome. In the

144 sample with the median number of total consensus reads, the average coverage depth across each

145 of the eight genes of interest exceeded 5,000 (Fig. 2B). After analyzing patterns in mutation

146 frequencies over time to eliminate other systematic biases (see Methods), we were able to track

147 the evolution and competition of 181 de novo mutations, including when many were present in

148 less than $0.1 \%$ of the cells in a population (Fig. 2C, Fig. 3). 
A

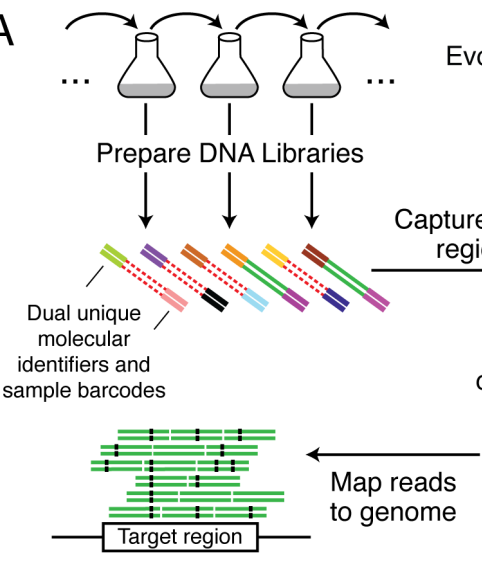

B
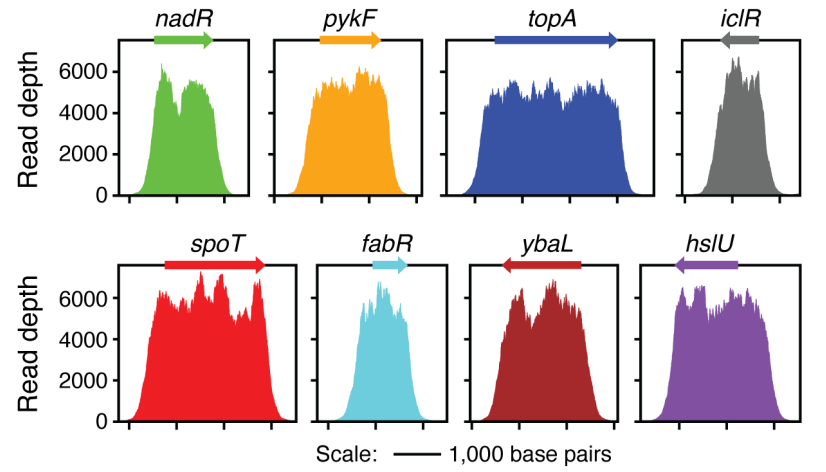

C

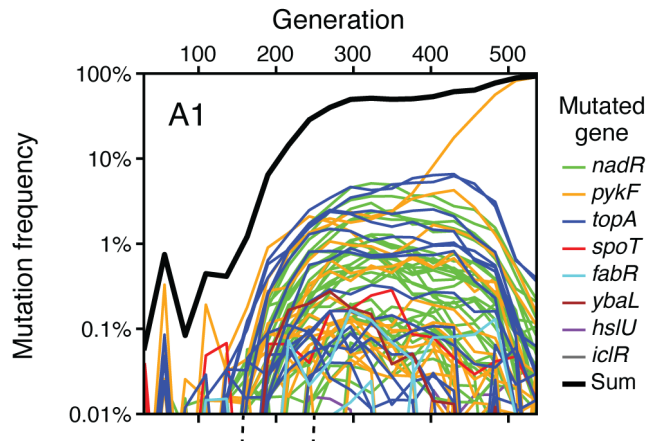

D

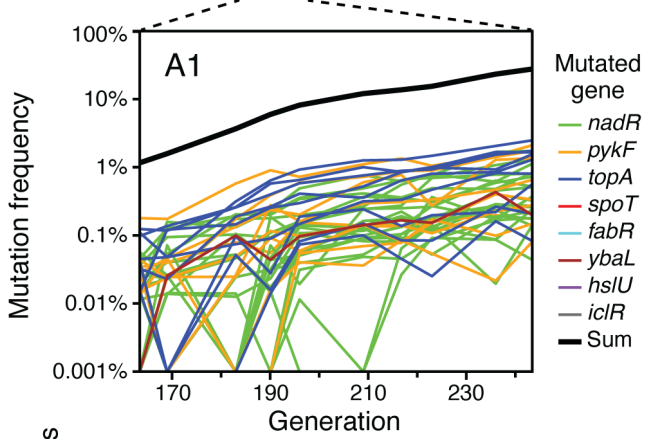

E

Figure 2. Profiling many beneficial mutations in the first selective sweep by deep sequencing. (A) Schematic of the deep sequencing approach. Genomic DNA is directly isolated from the $E$. coli populations and prepared for paired-end Illumina sequencing with sample barcodes and dual unique molecular identifiers (colored ends attached to red/green double stranded DNA). DNA fragments matching the targeted genome regions (green centers) are captured by probes (blue) bound to magnetic beads and other sequences are washed away (red centers). Reads in pairs that have the same dual unique molecular identifiers, which implies that they were PCR amplified during library preparation from the same original genomic DNA fragment, are used to construct consensus reads to eliminate sequencing errors. Consensus reads are mapped to the reference genome to call sequence variants. (B) Enrichment of reads mapping to eight genes known to be early targets of selection in this environment from the long-term evolution experiment. The final coverage depth of consensus reads in and around these genes is shown for a typical sample (population A7 at 500 generations). (C) Frequency trajectories for mutations in the eight targeted genes as well as the sum total frequency in population A1 over the complete time course of the evolution experiment. When a mutation was not detected at a time point, its trajectory is shown as passing through a frequency of $0.0001 \%$ (outside of the plot bounds). (D) Mutation frequency trajectories for population A1 during the window from 133 to 213 generations when mutations were first reaching detectable frequencies as they outcompeted the ancestral genotype. At time points when a mutation was not detected, its frequency is shown as $0.001 \%$ (at the bottom of the plot). (E) Estimated relative fitness of population A1 in each interval between the time points in the window time course. The frequency trajectories of all beneficial mutations in the initial sweep shown in D were used to jointly estimate the average fitness of the entire population from the deceleration in the rate of increase of the observed mutation trajectories as genotypes with beneficial mutations became common (see Methods). 
174 This fitness trajectory fit accounts for all cells in the population, regardless of whether they have 175 a mutation in the targeted genes or elsewhere in the genome. The red line is the maximum

176 likelihood estimate of the population fitness trajectory. The red shading around it shows $95 \%$

177 confidence intervals on this value in each interval. The black line shows the increase in fitness

178 estimated for a consensus model that was jointly fit to all mutations tracked in all six populations

179 for which sequencing data was collected in this window. The consensus population fitness

180 trajectory was used when estimating the fitness effects of individual mutations (see Methods) 
bioRxiv preprint doi: https://doi org/10.1101/2020.07.10.196832; this version posted January 5, 2021. The copyright holder for this preprint (which was not certified by peer review) is the author/funder, who has granted bioRxiv a license to display the preprint in perpetuity. It is made available under aCC-BY 4.0 International license.
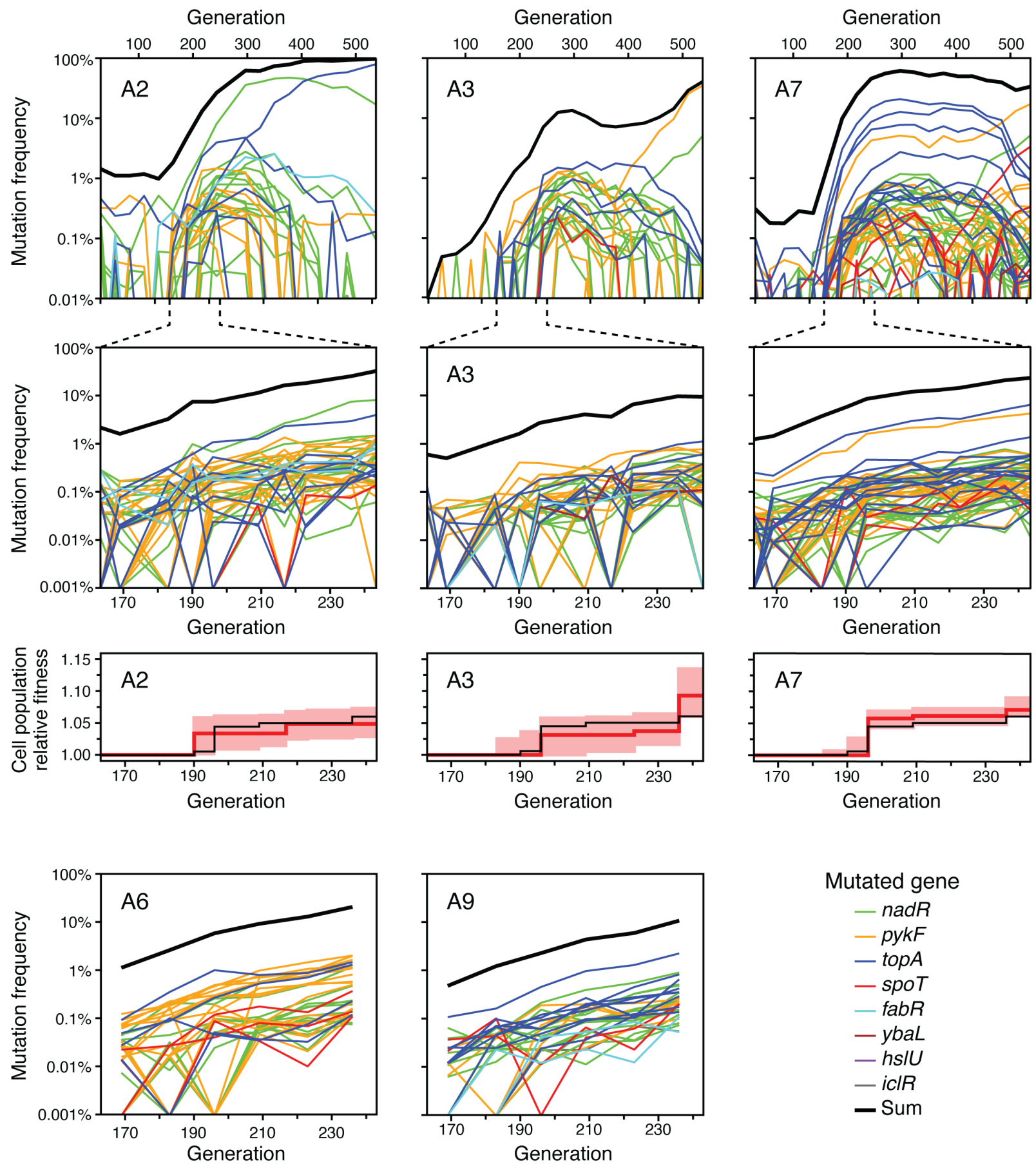

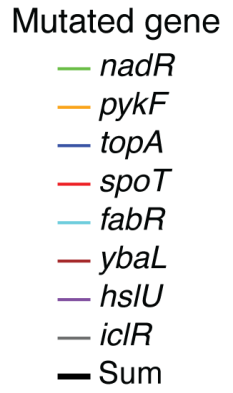
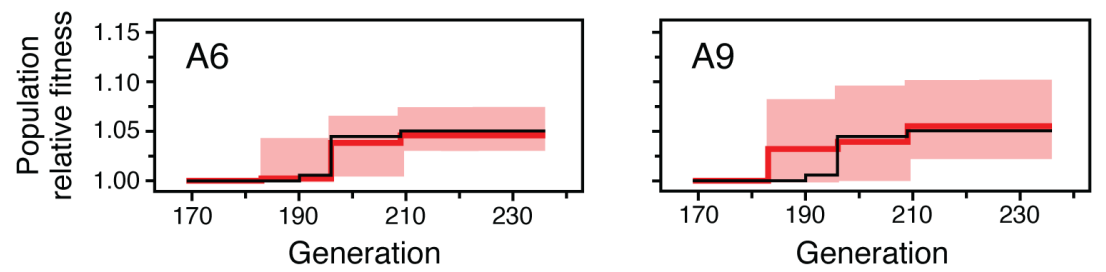

This population only

- Best fit $\quad 95 \% \mathrm{Cl}$

All populations together - Best fit 
182 Figure 3. Frequency trajectories of mutations in the remaining populations. The same plots 183 described in Figure 2C-E for population A1 are shown for populations A2, A3, and A7 (top three 184 sets of panels). For populations A6 and A9, sequencing was only performed at time points during 185 the selective sweep window so only the plots corresponding to Figure 2D-E are shown (bottom 186 two sets of panels).

Mutation trajectories in all four populations exhibited a burst of genetic diversity in the targeted genes followed by loss of this diversity. The initial dynamics are expected to be largely driven by new genotypes that each evolve a single beneficial mutation very early in the

191 experiment. If their descendants escape stochastic loss, they will gradually increase in frequency

192 over the first few hundred generations as they outcompete the ancestral genotype. Once the

193 population becomes dominated by these first-step mutants, their frequency trajectories plateau

194 because of clonal interference: they are now mainly competing against one another and are

195 relatively evenly matched. In populations $\mathrm{A} 1, \mathrm{~A} 2$, and $\mathrm{A} 7$, the total frequencies of the mutations

196 we identified sums to $50-62 \%$ at generation 270 , indicating that each population is mostly

197 composed of genotypes with a single mutation in one of the focal genes. We recovered less of

198 the initial beneficial mutation diversity in population A3 where this sum was only $13 \%$.

After around 300 generations, there is a steady decline in the frequencies of most mutations

200 in the eight targeted genes. At this point, new more-fit genotypes that have evolved begin to

201 exert their influence and displace the genotypes that we initially tracked. Many of the most

202 successful new genotypes are descended from cells that already had a mutation in one of the

203 targeted genes. In these cases, the original mutations serve as markers for the further expansion

204 of these subpopulations after a period during which their frequencies stagnate or decrease, but

205 the new beneficial mutations responsible for this further increase in fitness are outside of the 206 genomic regions we surveilled. The converse situation, in which a beneficial mutation in one of 207 the eight focal genes appears in a cell with an untracked beneficial mutation elsewhere in the 
208 genome, also occurs in a few cases. Most strikingly, a mutation in $p y k F$ that only appears after

209300 generations in population A3 rapidly increases in frequency and becomes dominant, strongly

210 suggesting that it appeared in a genetic background with a prior, unknown beneficial mutation.

\section{Selection coefficients can be inferred from initial mutation trajectories}

213 We next sought to calculate the fitness benefits of individual mutations by tracking how rapidly

214 their frequencies rose early in the experiment when they were largely competing versus the

215 ancestral genotype because all new mutations in the population were still rare. To that end, we

216 sequenced six populations at $\sim 13$-generation increments from 133 to 213 generations (Fig. 2D,

217 Fig. 3). We were able to track a total of 240 mutations as they gradually increased in frequency

218 during this critical time window. In the four populations we had already sequenced (A1, A2, A3,

219 and A7), these mutations included 120 of the 181 previously found in the complete time course

220 data spanning 500 generations and 54 additional mutations that were not detected in the complete

221 time course data. We also identified 66 new mutations in the window time courses of the two

222 populations that had not been previously sequenced (A6 and A9). Of these 240 mutations, 93.3\%

223 occurred in just three of the eight targeted genes: $n a d R, p y k F$, and top $A$ (Fig. 4A). 

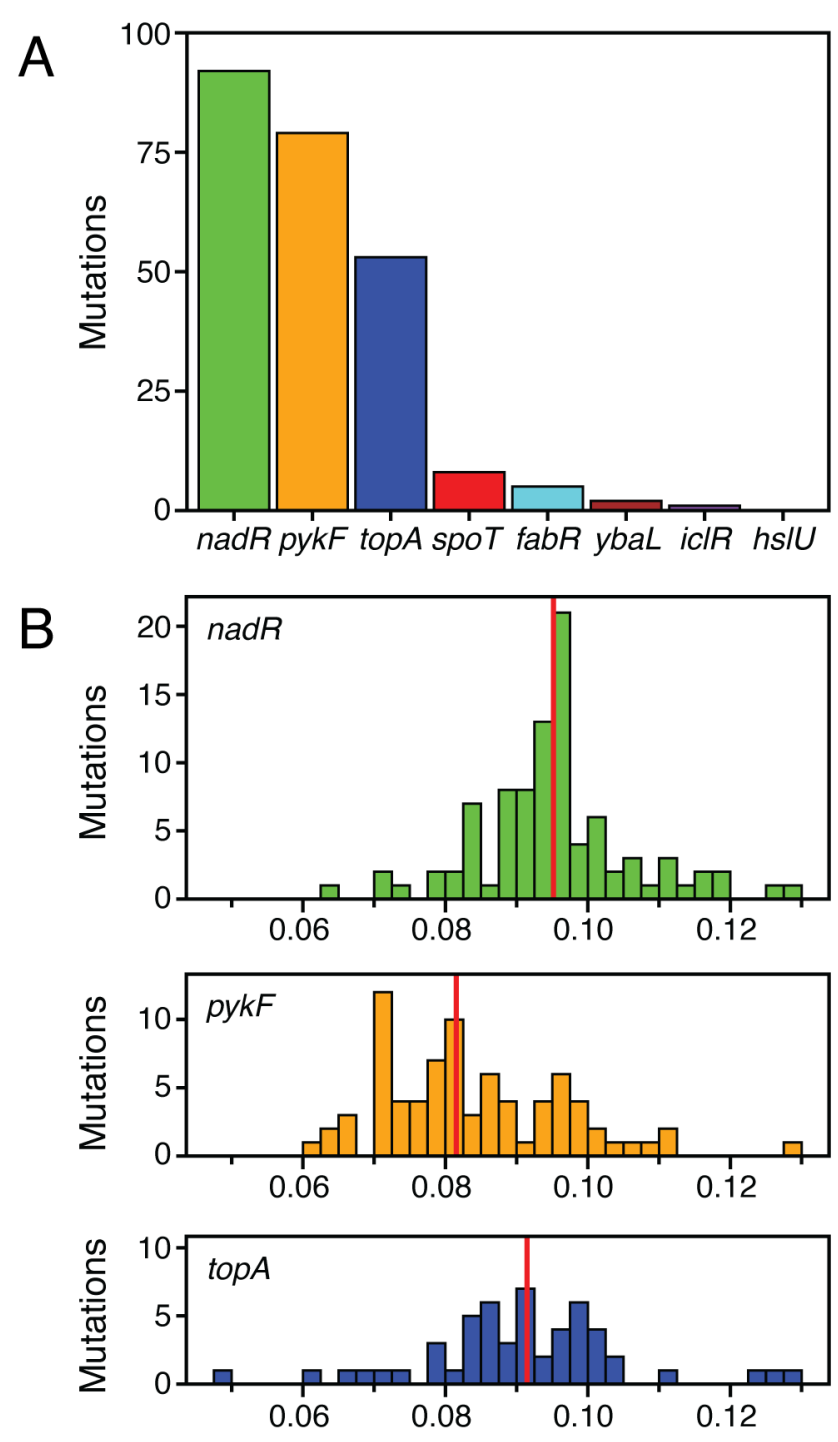

Fitness effect

Figure 4. Characteristics of beneficial mutations in the initial selective sweep. (A) Total number of beneficial mutations in each targeted gene identified in the window time courses from 133 and 213 generations for all six profiled E. coli populations. (B) Distribution of selection coefficients of beneficial mutations determined from the window time courses in the three genes that were the dominant targets of selection. Vertical red lines show the mean of each distribution.

We were able to estimate the fitness effect of each of the beneficial mutations predicted in the window time courses by fitting a binomial logistic model to the counts of reads supporting the variant versus reference sequences over time. In all populations, there is initially a log-linear increase in the frequency of each mutation as the first wave of evolved cells, nearly all of which are expected to have just one of these beneficial mutations, competes against a population that is 
236 still almost entirely cells with the ancestral genotype. Then, there is a deceleration in the rate at

237 which the frequencies of the new mutations increase around generation 196 that coincides with

238 the onset of clonal interference. Genotypes with beneficial mutations begin to make up a sizable

239 proportion of the population at this point, and making it necessary to account for how they are

240 increasingly competing against one another to estimate the fitness effect.

241 We accounted for clonal interference by adding a stepwise increase in the average fitness of

242 the entire cell population over time as an additional set of parameters to the binomial logistic

243 model (Fig. 2E, Fig. 3). That is, we estimated how the population fitness as a whole was

244 changing from the deceleration in the trajectories of the subset of mutations that we tracked in

245 the targeted genes. Because overall population fitness dynamics are highly reproducible from

246 population to population in the LTEE conditions (Lenski et al., 1991), we used one consensus

247 population fitness increase fit from all tracked mutations in all six sequenced populations to

248 correct our estimates of individual mutation fitness effects for clonal interference. Most of the

249 increase in population fitness occurs rapidly in a single step during the interval spanning 196-209

250 generations. This rapid change followed by stasis may seem at odds with the continuing increase

251 in the trajectories of many beneficial mutations. However, this type of stepwise increase is a

252 typical result of clonal dynamics in models and experiments (Gerrish and Lenski, 1998; Lenski

253 et al., 1991). It could reflect the influence of many mutations with small fitness effects, no one of

254 which reaches an observable frequency, peaking and then being outcompeted by the most

255 beneficial mutations that we are able to track, for example.

256 The mean fitness effect that we inferred for the 240 tracked mutations in all six populations

257 was $9.00 \%$ with a standard deviation of $1.33 \%$. Although the distributions of the fitness effects

258 estimated for mutations in $n a d R, p y k F$, and top $A$ overlap (Fig. 4B), there was a significant 
stratification among these genes. Mutations in $n a d R$ were $0.44 \%$ more beneficial than mutations

test). In turn, mutations in top $A$ were $0.70 \%$ more beneficial than those in $p y k F(p=0.00046$,

one-tailed Mann-Whitney U test). The fitness effects of the 16 mutations in the other genes

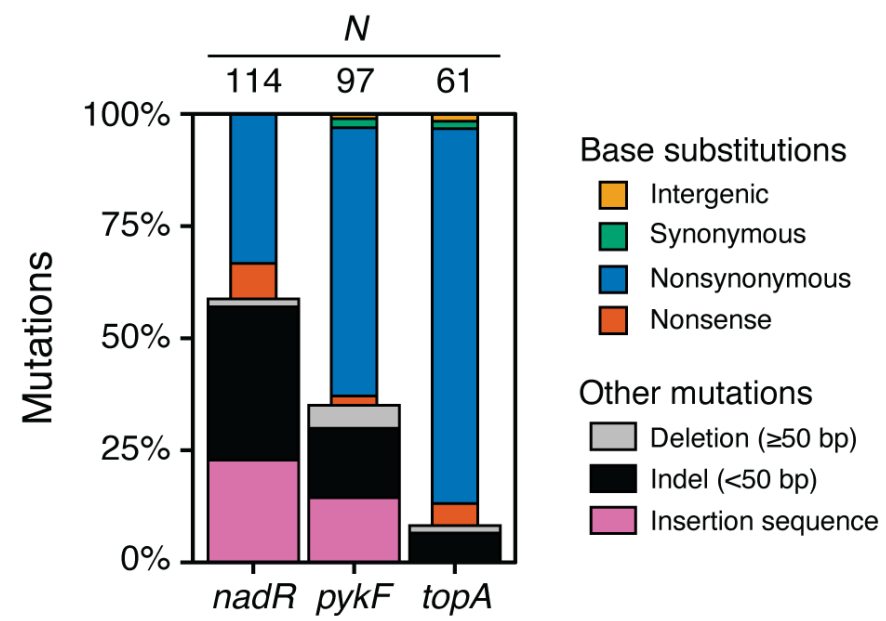

Figure 5. Spectra of early beneficial mutations in $n a d R, p y k F$, and top $A$. These three genes were the dominant targets of selection during the evolution experiment among the eight genes profiled for beneficial mutations. The bars include mutations identified in each of the six sequenced populations in its window time course, complete time course, or both. The total number of mutations identified in each gene is indicated above its column.

\section{Beneficial mutations reveal different signatures of selection on gene function}

276 Of the 301 mutations that we were able to track in complete or window time courses, 272 were

277 in the $n a d R, p y k F$, or topA genes. These large sets of highly beneficial mutations in these genes

278 gave us the statistical power to test for several signatures of molecular evolution to predict what

279 types of changes in the function of each gene improved E. coli fitness in this environment. Each 
280 of the three genes exhibited a distinct spectrum of beneficial mutations (Fig. 5). In some cases,

281 different types of mutations were also unevenly distributed throughout the sequences of these

282 three commonly hit genes and had noticeably different effects on bacterial fitness (Fig. 6A). 

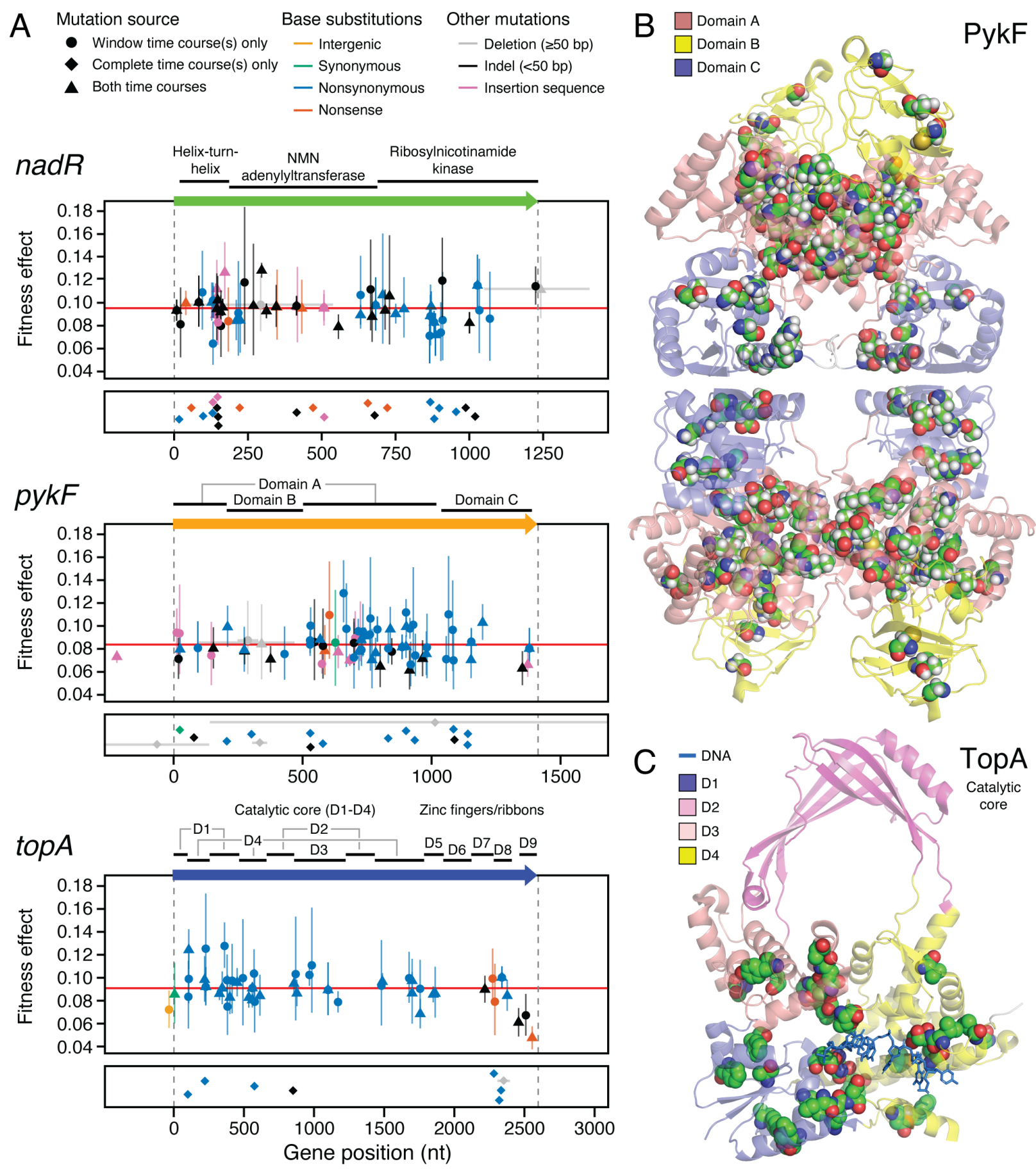

Figure 6. Mutations in the three genes that were the dominant targets of selection. (A) Nucleotide positions and properties of all mutations found in each of the three genes that were the dominant targets of selection during the evolution experiment. Colors represent the type of mutation. Symbols indicate whether each mutation was detected in the window time course, the complete time course, or both. The upper panel for each gene, shows the fitness effects estimated for mutations in that gene from the window time courses. Error bars are 95\% confidence limits.

291 When the same genetic change was detected in the window time courses for multiple populations the trajectories were combined to estimate one overall fitness value and confidence limit for all 
of those mutations (see Methods). Thus, the symbols and error bars for these mutations overlap in these cases. The reading frame of the gene is shown above this panel with protein domains labeled. Vertical dashed grey lines represent the start and end of each gene. Horizontal grey lines show the extent of large deletions within the pictured region. Horizontal red lines represent the average fitness effects for all mutations in a gene. The lower panel for each gene shows mutations that were only detected in the complete time courses and therefore do not have an estimated fitness effect. Symbols in this panel are randomly jittered in the vertical direction to improve their visibility. (B) Structural context of mutations in PykF. Sites of nonsymonymous mutations are highlighted by showing space-filling models of the substituted amino acid residues. All four subunits of the PykF homotetramer are shown. (C) Structural context of mutations in the catalytic core of TopA. Sites of nonsymonymous mutations are highlighted by showing space-filling models of the substituted amino acid residues. Only domains D1-D4 are present in the structure. The DNA strand interacting with TopA is shown as a stick model.

$$
\text { The E. coli nadR gene has three distinct functions related to NAD biosynthesis: (1) the N- }
$$

terminal domain is a helix-turn-helix that binds to DNA so that it can act as a negative

transcriptional regulator of NAD salvage and transport pathways; (2) the internal domain is an

NMN adenylyltransferase (Raffaelli et al., 1999); and (3) the C-terminal domain is predicted to have ribosylnicotinamide kinase activity (Kurnasov et al., 2003). Large deletions, frameshifts from small insertions or deletions (indels), insertions of transposable insertion sequence (IS) elements, and base substitutions creating stop codons dominate the nadR mutational spectrum (Fig. 5). These disruptive mutations, which are expected to result in complete loss of gene function, are significantly overrepresented versus nonsynonymous base substitutions in the first two domains of the gene compared to the remainder (13.7 odds ratio, $p=1.2 \times 10^{-8}$, one-tailed Fisher's exact test) (Fig. 6A). Yet, there was not a significantly greater selection coefficient for disruptive mutations compared to nonsynonymous mutations overall $(p=0.063$, one-tailed Mann-Whitney U test). These results suggest that complete inactivation of nadR yields the maximum benefit possible for a mutation in this gene. Disrupting all three of its distinct functions does not appear to be necessary for achieving this full benefit. Consistent with this prediction, deletion of $n a d R$ has been shown to be highly beneficial in the very similar environment of the LTEE (Barrick et al., 2009). 
Pyruvate kinase $1(p y k F)$ catalyzes the final step of glycolysis, transferring a phosphate group

324 from phophoenolpyruvate (PEP) to ADP to generate pyruvate and ATP. It is a key enzyme in

325 regulating glycolytic flux (Kochanowski et al., 2013; Siddiquee et al., 2004). We observed an

326 intermediate representation of disruptive mutations in $p y k F$, fewer than in nadR but more than in

327 top $A$ (Fig. 5). Interestingly, nonsynonymous base substitutions in $p y k F$ tend to have a larger

328 selection coefficient than disruptive mutations ( $p=0.00031$, one-tailed Mann-Whitney U test)

329 (Fig. 6A). This finding is in agreement with a study of various $p y k F$ alleles that arose in the

330 LTEE which found that nearly all $p y k F$ point mutations were more beneficial than deletion of the

$331 p y k F$ gene, both in the ancestor and in evolved genetic backgrounds (Peng et al., 2018). PykF

332 forms a homotetramer in which each polypeptide folds into three structural domains (Donovan et

333 al., 2016; Mattevi et al., 1995). The central domain A forms the active site at the interface with

334 domain B and the binding site for the allosteric effector fructose 1,6-bisphosphate at the interface

335 with domain $\mathrm{C}$. The nonsynonymous mutations that we observed are more concentrated than

336 expected in domain A versus the other structural domains based on their relative lengths in the

337 gene sequence $(p=0.0018$ one-tailed binomial test) (Fig. 6B). Overall, these results suggest that

338 complete inactivation of $p y k F$ is highly beneficial in the environment of our evolution

339 experiment, but mutations that alter its activity - likely in ways that reduce glycolytic flux — are

340 even more so. It has been suggested that reducing $p y k F$ activity is beneficial in the similar

341 glucose-limited conditions of the LTEE because this allows more PEP to be diverted to power

342 import of glucose into cells via the phosphotransfer system (Woods et al., 2006).

343 DNA topoisomerase I ( $\operatorname{top} A$ ) relaxes negative supercoiling introduced into the chromosome

344 by replication and transcription (Massé and Drolet, 1999). The mutations we observed in topA

345 are almost exclusively single-base substitutions (Fig. 5), suggesting that modulating the activity 
346 of this enzyme provides a fitness benefit. Indeed, complete loss of top $A$ function is lethal to $E$.

347 coli without compensatory mutations in DNA gyrase (Dinardo et al., 1982; Pruss et al., 1982).

348 The structure of E. coli TopA consists of four N-terminal domains (D1-D4) that make up the

349 catalytic core and five C-terminal zinc finger and ribbon domains (D5-D9) (Tan et al., 2015).

350 The few out-of-frame indels and the large deletion that we observe truncate TopA within

351 domains D7-D9, which interact with single-stranded DNA and RNA polymerase but are not

352 critical for catalysis. Considering only the catalytic core, we find that nonsynonymous mutations

353 are more concentrated in domains D1 and D4 versus D2 and D3 than expected from their relative

354 sizes $(p=0.00068$, one-tailed binomial test) (Fig. 6C). D1 and D4 together form the ssDNA

355 binding groove leading to the active site, and D1 also forms part of the active site at its interface

356 with D3 (Perry and Mondragón, 2003). Several base substitutions in topA have been shown to

357 increase positive supercoiling in evolved LTEE strains (Crozat et al., 2005, 2010). The exact

358 reason that this change in supercoiling is beneficial is unknown, but it may be linked to

359 increasing the expression of ribosomal RNAs (Crozat et al., 2005), altering gene regulation

360 responses to starvation or other stresses (Crozat et al., 2010), and/or increasing the expression of

361 genes in divergently transcribed operons (Houdaigui et al., 2019).

363 Recurrent beneficial mutations do not have greater fitness effects

364 We observed many examples of exact genetic parallelism. That is, the same mutation occurred

365 and reached high frequency in different experimental populations. Each of these E. coli

366 populations was founded from single cells, so we can conclude that these recurrent genetic

367 changes are due to independent mutational events. We observed a total of 252 distinct genetic

368 changes across all eight profiled genes and 31 of these were found in more than one population. 
369 While no single genetic change was detected in all six populations, 2, 2, 8 and 19 changes were

370 detected in 5, 4, 3, and 2 populations, respectively. Most of these were in the three genes that

371 were the main targets of selection (nadR, $p y k F$, and top $A$ ), but one that occurred in three

372 populations was in $f a b R$. These mutations may be recurrent because they have a higher fitness

373 benefit than other mutations, occur at a higher rate than other mutations, are more easily detected

374 in the sequencing data, or due to some combination of these factors. We had a fitness estimate

375 for each of the 31 recurrent mutations from tracking cells with that genetic change in at least one

376 of the window time courses and had 167 fitness measurements for mutations that were observed

377 in only one population. The recurrent mutations had a $0.12 \%$ greater fitness effect, on average,

378 compared to the singleton mutations, but this difference was not significant $(p=0.25$, one-tailed

379 Mann-Whitney U test). Thus, it is unlikely that many cases of exact genetic parallelism are due

380 to these mutations being more beneficial than others in our dataset.

\section{Discussion}

383 We examined bacterial evolution during the initial stages of clonal competition when there is a

384 burst of beneficial genetic diversity as many new subpopulations with different mutations evolve

385 and begin to displace the ancestral genotype. We focused on eight genes known to accumulate

386 adaptive mutations in the $>70,000$ generation Lenski long-term evolution experiment (LTEE)

387 with $E$. coli that used nearly the same environment as our experiments. The only difference was

388 that we added four times as much of the limiting nutrient (glucose). By combining Illumina

389 sequencing using unique molecular identifiers for error correction, hybridization-based capture

390 of DNA encoding these genes, and dense temporal sampling, we were able to identify more

391 beneficial mutations and track them at much lower frequencies than is possible with standard 
metagenomic sequencing. We detected a total of 301 mutations in the focal genes: 181 in the

populations and two others, with 120 mutations overlapping between the two sets.

By densely sampling and deeply sequencing E. coli populations, we were able to characterize

401 sampling scheme. Considering both the complete and window time courses we characterized 241

413 LTEE, but they typically occur later (often within the first 2,000 to 10,000 generations) (Good et 
415 were in $t o p A, p y k F$, and $n a d R$, but we also found multiple mutations that were similarly

416 beneficial in spoT, fabR, and $y b a L$. Mutations in $n a d R$ were more widespread than expected in

417 our experiment and may be more likely to completely disrupt its function than beneficial alleles

418 that evolve in the LTEE (Ostrowski et al., 2008). Mutations in spoT and fabR were rarer than

419 expected from the LTEE. One possible explanation for these slight differences is the increased

420 concentration of glucose in our experiment compared to the LTEE. These minor deviations are

421 also reminiscent of how changing a different aspect of the environment (temperature) re-focuses

422 the mutations of largest benefit that succeed early onto different subsets of genes, nearly all of

423 which eventually accumulate beneficial mutations later in the LTEE environment, in related

424 evolution experiments (Deatherage et al., 2017; Tenaillon et al., 2012). Alternatively, we cannot

425 rule out that mutations with similar fitness effects are still possible in the rarely mutated genes

426 but that there are so many fewer possibilities for these mutations that they were not often

427 sampled in our evolving populations. Despite these subtle differences between the LTEE and our

428 experiment, we were still able to account for majority of the genetic variation present in three of

429 the four populations that we profiled over the entire 500 generations by analyzing evolution in

430 the eight candidate genes.

431 We also wanted to understand to what extent we gained early warning of driver mutations by

432 deeply profiling evolution in genes we expected to be under strong selection. In general, we were

433 able to begin tracking most mutations when they were above a frequency of $0.01 \%$. This level of

434 profiling enabled us to first detect mutations an average of 69,150 , and 290 generations before

435 they surpassed frequencies of $0.1 \%, 1 \%$, and $5 \%$, respectively. Under the conditions of our

436 experiment these intervals take roughly 10, 23, and 44 days, respectively; so, even though we

437 made these predictions retrospectively, there would have been sufficient time to complete the 
438 DNA isolation, library preparation, sequencing, and analysis steps quickly enough for this

439 approach to give early warning of specific genetic variants driving evolution of these

440 populations. The amount of lead time becomes disproportionately longer at higher frequencies

441 due to clonal interference between beneficial mutations. The chances and timescales of earlier

442 detection are expected to increase even more when there are ecological interactions or spatial

443 structure that further slow the takeover of new variants, as has been demonstrated and discussed

444 in other microbial evolution experiments (Baym et al., 2016; Frenkel et al., 2015; Traverse et al., 445 2013).

446 A further prediction is that the genes in which we observe early, but unsuccessful beneficial

447 mutations will sustain mutations again and again until they are successful in a population's

448 evolutionary future. This prediction is limited by the nature of epistatic interactions. In the LTEE

449 and other microbial evolution experiments, diminishing returns epistasis dominates between

450 beneficial mutations in different genes (Chou et al., 2011; Khan et al., 2011; Kryazhimskiy et al.,

451 2014; Wei and Zhang, 2019; Wiser et al., 2013). That is, mutations in one gene that improve the

452 fitness of the ancestor tend to still be beneficial to evolved genotypes containing beneficial

453 mutations in other genes, just less so than when those other mutations are not present.

454 Subpopulations with mutations in both $n a d R$ and $p y k F$ evolve by 20,000 generations in all 12

455 LTEE populations, and cells that also contain a mutation in top $A$ are found in six of the LTEE

456 populations at this point (Tenaillon et al., 2016). By this time, mutations in $y b a L$ and spoT are

457 also found in nine and six LTEE populations, respectively. So, for five of the six genes in which

458 we detected multiple mutations in the initial burst phase, it is likely that nearly all of them would

459 have eventually accumulated beneficial mutations if we continued our experiment. 
The other three genes $(f a b R, i c l R$, and $h s l U)$ likely represent other scenarios. Mutations in

461 fabR transiently appear within the first 2,000 generations of the LTEE (Deatherage et al., 2015).

462 They interact unfavorably with beneficial mutations in spoT and other genes, such that a fabR

463 mutation essentially precludes further adaptation by mutating the other set of genes and vice-

464 versa (Deatherage et al., 2015; Woods et al., 2011). We detected 9 mutations in fabR, which was

465 more than the five we observed in $y b a L$. However, we predict that $f a b R$ mutations are unlikely to

466 re-emerge and be successful in the future of these populations because of their negative

467 interactions with other beneficial mutations. On the other hand, we detected only a single

468 mutation in $i c l R$ and a single mutation in $h s l U$. Of the 12 LTEE populations, 11 have sizable

469 subpopulations with mutations in $i c l R$ and 11 have mutations in $h s l U$ by 20,000 generations,

470 which makes them more common than mutations in spoT and $y b a L$ in the long run. Therefore,

471 mutations in $i c l R$ and $h s l U$ appear to either require the presence of mutations in other genes to

472 become highly beneficial or may not be able to experience any mutations that are beneficial

473 enough to make them competitive early on in our experiment.

474 The nature of epistasis and the limits that it imposes on predicting the future evolution of a

475 cell population could be further probed using our approach in several ways. One could repeat the

476 evolution experiment beginning with genotypes containing different first-step beneficial

477 mutations as starting points to more finely map the fitness landscape. One could also interrogate

478 the diverse collections of cells containing different beneficial alleles that we have evolved, by

479 taking the 150-generation populations and further evolving them under different conditions to

480 map genotype by environment effects, for example. Such experiments might also reveal latent

481 beneficial mutation in other genes (e.g., $i c l R$ and $h s l U$ ) that were able to outcompete the ancestor

482 in our experiment but remained below the detection limit because they were not as beneficial as 
483 mutations in top $A, p y k F$, and $n a d R$ in this environment. There is precedent for changes in the

484 environment deflecting selection to different subsets of the same genes. In an offshoot of the

485 LTEE that began with a clone that had $s p o T$, top $A$, and $p y k F$ mutations, selection was focused on

486 either $h s l U$, iclR, or nadR depending on changes in temperature (Deatherage et al., 2017).

Alternative and complementary methods exist for deeply profiling the evolutionary

possibilities inherent in the fitness landscape of a cell, i.e., its evolvome. We tracked spontaneous

specifically describe as the adaptome (Ryall et al., 2012). Amplicon sequencing can also capture

mutations in a subset of the genome with deep coverage (Chubiz et al., 2012; Fischer et al., samples (Newman et al., 2014, 2016). Tracking the frequencies of barcoded cells and their progeny has been used to characterize the statistical properties of much larger collections of

499 naturally occurring beneficial mutations and when they are much rarer within cell populations

500 (Levy et al., 2015; Venkataram et al., 2016). However, one must barcode individuals in the

501 population to apply this method, which may be difficult in certain cell types or in clinical

502 samples, and additional genome sequencing after an experiment is completed is required to

503 discover the identities of the beneficial mutations linked to barcodes. Other methods such as

504 deep-mutational scanning (Fowler and Fields, 2014) or CRISPR-enabled trackable genome

505 engineering (Garst et al., 2017) can simultaneously interrogate large libraries of mutants to map 
506 evolvomes. However, since they artificially construct variant libraries, they do not necessarily

507 provide information about which genetic variants are accessible by spontaneous mutations and

508 would therefore be expected to contribute the most to a cell's adaptome.

509 Exhaustively mapping paths that clonal evolution is likely to follow is of particular interest

510 and utility in systems that evolve repeatedly from a defined starting point. These range from

511 bioreactors that are seeded with the same strain in different production runs to lung infections in

512 cystic fibrosis patients that start from similar, but not identical, opportunistic pathogens. The

513 ability to identify mutations in key genes while they are still very rare may also be used to

514 improve the early detection of emerging drug resistance in other human infections and cancer.

515 The evolutionary dynamics will be more complex in many of these systems, but they may also

516 unfold more slowly. For example, biofilm formation and the necessity of invading already

517 colonized niches will slow the dynamics of competition. This potentially makes the therapeutic

518 window for detecting incipient evolution by profiling the adaptome even greater.

520 Materials and Methods

521 Evolution experiment

522 Strains and growth conditions are derived from the Lenski long-term evolution experiment

523 (Lenski and Travisano, 1994; Lenski et al., 1991). Nine colonies of E. coli B strain REL606 and

524 nine of strain REL607 were selected at random. Each was used to inoculate a separate flask

525 containing $10 \mathrm{~mL}$ of Davis Minimal (DM) media supplemented with $100 \mu \mathrm{g} / \mathrm{L}$ glucose

526 (DM100). This is a slightly higher concentration of glucose than the $25 \mu \mathrm{g} / \mathrm{L}$ glucose (DM25)

527 used in the LTEE, but still well below the $\sim 1000 \mu \mathrm{g} / \mathrm{L}$ concentration at which nutrients other

528 than glucose begin to limit growth in this medium. We used this higher glucose concentration to 
529 ensure we had a sufficient number of cells for sequencing and archiving. These initial cultures

530 were grown overnight at $37^{\circ} \mathrm{C}$ with orbital shaking over a one-inch diameter at $120 \mathrm{RPM}$.

531 Approximately 30 generations of growth occurred starting from the initial single cell that gave

532 rise to each colony until saturation of these cultures. Next, $10 \mathrm{~mL}$ of fresh DM100 were

533 inoculated with $50 \mu \mathrm{L}$ of one REL606 culture and $50 \mu \mathrm{L}$ of one REL607 culture for overnight

534 growth in the same conditions. The remaining culture was archived at $-80^{\circ} \mathrm{C}$ with $2 \mathrm{~mL}$

535 dimethyl sulfoxide (DMSO) added as cryoprotectant. Daily transfer of $100 \mu \mathrm{L}$ of overnight

536 culture to $10 \mathrm{~mL}$ of fresh DM100 and archival of the remaining culture volume in the same way

537 continued through 75 daily transfers. Periodically $1 \mu \mathrm{L}$ of culture was diluted 10,000-fold in

538 sterile saline and plated on tetrazolium arabinose (TA) agar to allow growth of $\sim 200$ colonies.

539 REL606 and REL607 differ by a mutation in an arabinose utilization gene that makes REL606

$540\left(\mathrm{Ara}^{-}\right)$colonies red and REL607 $\left(\mathrm{Ara}^{+}\right)$colonies pink (Lenski et al., 1991). The ratio of red to

541 pink colonies was used to monitor these populations for selective sweeps (Hegreness and

542 Kishony, 2007; Woods et al., 2011).

\section{DNA isolation and library preparation}

545 Genomic DNA (gDNA) was isolated from frozen population samples by first thawing each 15

$546 \mathrm{~mL}$ conical tube on ice. Of the $\sim 12 \mathrm{~mL}$ total volume of culture plus cryoprotectant, $1.2 \mathrm{~mL}$ was

547 transferred to a $2 \mathrm{~mL}$ cryovial and refrozen. The remaining $\sim 10.8 \mathrm{~mL}$ was centrifuged at $6,500 \times$

$548 \mathrm{~g}$ at $4^{\circ} \mathrm{C}$ for 15 minutes. The resulting cell pellets were transferred with a volume of remaining

549 solution to $1.7 \mathrm{~mL}$ Eppendorf tubes. Then, gDNA was isolated using the PureLink Genomic

550 DNA Mini kit (Life Technologies). For each sample, $1 \mu \mathrm{g}$ of gDNA was randomly fragmented

551 on a Covaris S2 focused-ultrasonicator. 
Illumina libraries were constructed using the Kappa Biosystems LTP Library Preparation Kit with the following modifications. End-repaired, fragmented DNA was T-tailed (rather than A-

554 tailed) in a $50 \mu 1$ reaction including $10 \mathrm{mM}$ dTTP and 5 units of Klenow fragment, exo ${ }^{-}$(New

555 England Biolabs). Illumina adapters containing 12-base unique molecular identifiers were ligated

556 to the T-tailed fragments as previously described (Schmitt et al., 2012), except full-length

557 adapter sequences containing unique external sample barcodes were directly ligated to the T-

558 tailed dsDNA inserts to reduce the risk of cross-contamination between samples. The full list of

559 DNA sequence adaptors used is provided in Table S1.

561 Probe design and target capture

562 Oligonucleotide probes consisting of 60-base xGen Lockdown probes (Integrated DNA

563 Technologies) were designed to tile across each of the eight genes of interest including upstream

564 promoter elements. Probes for each gene were compared to the entire E. coli B strain REL606

565 reference genome (GenBank: NC_012967.1) (Jeong et al., 2009) using BLASTN (Camacho et

566 al., 2009). The starting positions of all probes in a set were shifted by one base at a time until

567 every probe had only a single significant predicted binding location (match with E-value $<$

$5682 \times 10^{-5}$ ). The sequences of the final set of 242 probes are provided in Table S2.

569 Capture was performed using a SeqCap EZ Exome Enrichment kit v3.0 (NimbleGen) with

570 several modifications to the protocol. First, 18 to 20 population samples with different sample

571 barcodes were pooled together in a single capture reaction that contained a total of $1 \mu \mathrm{g}$ of

572 library DNA from all samples, $1 \mathrm{mmol}$ of a universal blocking oligo, and $1 \mathrm{mmol}$ of a degenerate

573 sample barcode blocking oligo. The sequences of these blocking oligos are provided in Table

574 S3. Second, after hybridization for $72 \mathrm{~h}$, DNA fragments hybridized to the biotinylated probes 
were recovered using MyOne Streptavidin C1 Dynabeads (Life Technologies). Third, a final 8cycle PCR step was performed with HiFi Hotstart DNA Polymerase (Kappa Biosystems).

\section{Sequencing and read processing}

Paired-end 101- or 125-base sequencing of the final libraries was performed on an Illumina HiSeq 2000 at the University of Texas at Austin the Genome Sequencing and Analysis Facility (GSAF). Read sequences have been deposited into the NCBI Sequence Read Archive (PRJNA601748). Raw reads were used to generate Consensus Sequence Reads (CSR) using custom Python scripts that carried out the following steps. First, the beginning of each read was evaluated for the presence of the expected 5'-end tag, consisting of a random 12-base unique molecular identifier (UMI) followed by four fixed bases $\left(5^{\prime}-\mathrm{N}_{12} \mathrm{CAGT}\right)$. Read pairs lacking the correct 5 '-end tag on either read were discarded. Across all samples, $80.2 \%$ of read pairs had both UMIs. For remaining read pairs, the UMIs found on each end were concatenated to create a 24-base dual-UMI that uniquely identifies the original DNA fragment that was amplified and sequenced. To group all reads corresponding to the same initial DNA molecule, a FASTA file of all dual-UMIs was used as input into the ustacks program from the Stacks software pipeline (Version 1.48) (Catchen et al., 2013) with the following options: a single read was sufficient to seed a stack, a single mismatch within the dual-UMI was allowed in assigning a read to a stack, secondary reads and haplotypes were disabled, and stacks with high coverage were preserved. Then, CSRs were generated for all dual-UMI groups sequenced at least twice by taking the straight consensus of all reads that were merged into that stack. If no base exceeded $50 \%$ frequency at a given position in this set of reads, then that base was set as unknown $(\mathrm{N})$. Of the read pairs with valid dual-UMIs, $41.6 \%$ were incorporated into consensus reads across all 
samples. The average number of dual-UMI read pairs utilized to create each consensus read was $16.9 \%$.

601

\section{Variant calling}

603 We used the breseq pipeline (Barrick et al., 2014; Deatherage and Barrick, 2014; Deatherage et

604 al., 2015) (version 0.26.0) to call single-nucleotide variants (SNVs) and structural variants (SVs)

605 from the CSRs. We divided the genome sequence of the ancestral E. coli REL606 strain into two 606 types of reference regions for mapping in this analysis. The eight regions of the genome tiled

607 with probes - extended with hundreds of bases of flanking sequence on both sides - were input 608 as "targeted" sequences, and the remainder of the genome with the identical eight regions 609 masked to degenerate $\mathrm{N}$ bases was supplied as a "junction-only" reference (to which reads are 610 mapped without variant calling). All 116 samples were analyzed using breseq in polymorphism

611 prediction mode with all bias, minimum allele frequency, and read-count filters disabled.

612 Evidence items in the Genome Diff (GD) files for all samples were combined using the gdtools 613 utility program to generate a single merged GD file with each piece of evidence listed a single

614 time, regardless of how many times it was detected in different samples. We then re-ran breseq 615 using the same parameters except that this GD file was supplied as an input user-evidence file to 616 force output of variant and reference information for these putative variants in every sample.

617 Then, we extracted the number of variant reads supporting each putative variant allele and the 618 total number of reads at that reference location from the GD file output by breseq. Subsequent 619 statistical tests and fitting steps were performed in R (version 4.0.0) (R Core Team, 2016) using 
620 the ggplot2 package for data visualization (Wickham, 2016). Scripts and data files for this

621 analysis are available in GitHub (https://github.com/barricklab/adaptome-capture).

622 Since this original analysis was conducted at the level of breseq evidence (i.e., single

623 columns of read pileups on the reference genome or instances of new sequence junctions), we

624 next merged sets of observations that were consistent with a single mutational event when they

625 also had frequency trajectories that tracked together. To identify these candidates for merging,

626 we analyzed each of the six window (generation 133 to 213) and four complete (generation 0 to

627 500) time courses separately. We only considered mutations that exceeded a threshold frequency

628 of $0.03 \%$ at some time during each time course as candidates for merging.

629 Read alignment (RA) evidence items were merged when they were located within 6 base

630 pairs of one another and within a normalized Canberra distance of 0.1 between the vectors of

631 their frequency observations across all of the time points in a dataset. All RA evidence pairs of

632 this kind were found to co-occur in the same sequencing reads. For these cases, the read counts

633 for the first linked mutation were used to represent the entire event. For example, if a deletion of

634 three base pairs was predicted by missing bases at positions $\mathrm{x}, \mathrm{y}$, and $\mathrm{z}$; then the frequency of

635 missing the first base (x) was assigned to the entire three-base deletion mutation.

636 For new junction (JC) evidence we performed the same merging procedure but allowed

637 linked mutations to be within a larger window of 20 base pairs and within a normalized Canberra

638 distance of 0.5 . JC pairs passing these criteria were only merged if they were also consistent with

639 an IS-element insertion in terms of their relative orientation and spacing. In this case the variant

640 and total read counts were added together for the two different junctions, as the junctions on each

641 side of the inserted IS element provide independent information for estimating the frequency of

642 this type of mutation. We allowed unpaired JC evidence passing the filters to also predict IS 
643 element mutations. This situation may indicate that there was an IS-mediated deletion between

644 an element that inserted within the gene and another element from the same family located

645 outside of the targeted region or more complex chromosomal rearrangements involving a newly

646 inserted IS element (Raeside et al., 2014).

648 Time course filtering and fitness effect estimation

649 After merging evidence of genetic variants into lists of putative mutations, we further eliminated

650 some of these from consideration using several filtering steps. For the complete time courses, we

651 first required that non-zero frequencies be observed for a mutation in samples from at least two

652 different time points. We next applied a filter to eliminate spurious variants that can be

653 recognized as arising from systematic sequencing or alignment errors because they do not exhibit

654 the correlated changes in frequency over time expected for the frequency trajectories of real

655 mutations (Lang et al., 2013). Specifically, we required that the time-series of estimated

656 frequencies for a mutation over all analyzed time points have an autocorrelation value $\geq 0.55$.

657 For the window time courses, we eliminated putative mutations for which there was great

658 uncertainty in the estimated fitness effect or evidence that its trajectory reflected multiple

659 beneficial mutations occurring in the same genome. Specifically, we required that a mutation

660 was first observed at generation 196 or earlier and that its estimated frequency was $\geq 10^{-4}$ in

661 every sample that was sequenced from generation 223 to 243 . Then, we fit a binomial logistic

662 model with slope and $y$-intercept terms to the time courses of counts of variant and reference

663 (total minus variant) observations for each mutation. We used a negative offset in the model of

664 the number of generations up to each time point so that the slope represents one plus the

665 selection coefficient that is characteristic of the subpopulation with that mutation. We filtered out 
666 any mutations for which this fit had an $\mathrm{AIC}<200$, a $p$-value for the slope differing from zero of

$667>0.005$, or a $y$-intercept $<-20$. The fitness effects that we report for mutations are the selection

668 coefficients fit from the model divided by the natural logarithm of two so that they are expressed

669 per generations of binary cell division. One plus the fitness effect is the relative fitness of a cell

670 with that mutation. These values can be directly compared to experimental measurements of

671 relative fitness and mutation fitness effects made using co-culture competition assays (Lenski et

672 al., 1991; Tenaillon et al., 2016).

673 This procedure for determining fitness effects assumes that the trajectories reflect

674 competition purely against the ancestral strain. However, we detected a consistent deviation from

675 linearity for all mutation trajectories in the window time courses after generation 196. The rates

676 at which the frequencies of all mutations were increasing decelerated, indicating that the overall

677 population fitness had improved to a degree that it reduced their effective advantage versus their

678 competitors. To account for this change we fit additional parameters defining a stepwise increase

679 in the average relative fitness of the population within each interval between sequenced samples

680 from generation 196 onward. The increase in population fitness reduces the effective time basis

681 used in the model to determine the slope to the number of generations in each interval divided by

682 the average relative fitness during that interval. We determined the population fitness values that

683 minimized the AIC of this modified binomial logistic model. The figures show the best stepwise

684 increases in population fitness between the sequenced time points from generation 196 onward

685 fitting to the trajectories of all mutations in a given population at the same time. We performed

6861000 bootstrap resamplings of the mutations in each population to estimate $95 \%$ confidence

687 intervals on the estimated population fitness values in each interval for that population. 
We combined information across multiple populations in two ways to further improve the

690 the stepwise population fitness increases for each population considered alone. Because the

691 actual population fitness trajectories of all populations are expected to be highly similar to one

692 another, we fit a consensus stepwise increase in population fitness over time that best improved

693 the fits for all mutations from all populations. Second, we observed 27 cases in which the exact

694 same change in a gene's sequence was observed and passed our filtering criteria in the window

695 time courses of multiple experimental populations. Because each population was started from

696 single cells, we can be sure that these are independent observations of the same mutation.

697 Therefore, we fit one consensus fitness effect (slope) for each of these recurrent mutations across

698 all populations. We still allowed the $y$-intercept for each of these mutations to vary from

699 population to population because this parameter is related to how early the mutation evolved,

700 which is expected to be different in each replicate population.

701

702 Protein structure analysis

703 Structural domains in NadR, PykF, and TopA were defined according to UniProt and papers

704 reporting x-ray crystal structures. Mutations in PykF were mapped onto Protein Data Bank

705 structure 4YNG (Donovan et al., 2016). Mutations in TopA were mapped onto Protein Data

706 Bank structure 1MW8 (Perry and Mondragón, 2003). Protein structures were visualized using

707 Pymol v2.3.5 (Schrödinger LLC).

708

709 Acknowledgements 
710 The authors acknowledge the Texas Advanced Computing Center (TACC) at The University of

711 Texas at Austin for providing high-performance computing resources.

712

\section{Supporting Information}

714 Table S1 Adapter sequences used in DNA library preparation

715 Table S2 Sequences of pulldown probes

716 Table S3 Blocking oligos used to limit read-to-read binding during pulldown

717

718 Author contributions

719 Conceptualization: DED JEB.

720 Data Curation: DED JEB.

721 Funding Acquisition: DED JEB.

722 Investigation: DED.

723 Methodology: DED JEB.

724 Software - DED JEB.

725 Supervision - JEB.

726 Visualization: DED JEB.

727 Writing - Original Draft Preparation: DED JEB.

728 Writing - Review \& Editing: DED JEB.

\section{References}

Bainbridge, M.N., Wang, M., Burgess, D.L., Kovar, C., Rodesch, M.J., D’Ascenzo, M.,

733 Kitzman, J., Wu, Y.-Q., Newsham, I., Richmond, T. a, et al. (2010). Whole exome capture in

734 solution with $3 \mathrm{Gbp}$ of data. Genome Biol. 11, R62.

735 Barrick, J.E. (2020). Limits to predicting evolution: insights from a long-term experiment with 
Escherichia coli. In Evolution in Action: Past, Present and Future, W. Banzhaf, B.H.C. Cheng, K. Deb, K.E. Holekamp, R.E. Lenski, C. Ofria, R.T. Pennock, W.F. Punch, and D.J. Whittaker, eds. (Cham: Springer), pp. 63-76.

Barrick, J.E., and Lenski, R.E. (2009). Genome-wide mutational diversity in an evolving population of Escherichia coli. Cold Spring Harb. Symp. Quant. Biol. 74, 119-129.

741 Barrick, J.E., and Lenski, R.E. (2013). Genome dynamics during experimental evolution. Nat.

742 Rev. Genet. 14, 827-839.

743 Barrick, J.E., Yu, D.S., Yoon, S.H., Jeong, H., Oh, T.K., Schneider, D., Lenski, R.E., and Kim, J.F. (2009). Genome evolution and adaptation in a long-term experiment with Escherichia coli. Nature 461, 1243-1247.

Barrick, J.E., Colburn, G., Deatherage, D.E., Traverse, C.C., Strand, M.D., Borges, J.J., Knoester, D.B., Reba, A., and Meyer, A.G. (2014). Identifying structural variation in haploid microbial genomes from short-read resequencing data using breseq. BMC Genomics 15, 1039. Spatiotemporal microbial evolution on antibiotic landscapes. Science 353, 1147-1151.

Camacho, C., Coulouris, G., Avagyan, V., Ma, N., Papadopoulos, J., Bealer, K., and Madden, T.L. (2009). BLAST+: architecture and applications. BMC Bioinformatics 10, 421.

753

754

755

756

757

758

759

760

761

762

763

764

765

766

767

768

769

770

771

772

773

774

Catchen, J., Hohenlohe, P.A., Bassham, S., Amores, A., and Cresko, W.A. (2013). Stacks: an analysis tool set for population genomics. Mol. Ecol. 22, 3124-3140.

Chou, H.-H., Chiu, H.-C., Delaney, N.F., Segrè, D., and Marx, C.J. (2011). Diminishing returns epistasis among beneficial mutations decelerates adaptation. Science 332, 1190-1192.

Chubiz, L.M., Lee, M.-C., Delaney, N.F., and Marx, C.J. (2012). FREQ-Seq: a rapid, costeffective, sequencing-based method to determine allele frequencies directly from mixed populations. PLoS ONE 7, e47959.

Crozat, E., Philippe, N., Lenski, R.E., Geiselmann, J., and Schneider, D. (2005). Long-term experimental evolution in Escherichia coli. XII. DNA topology as a key target of selection. Genetics 169, 523-532.

Crozat, E., Winkworth, C., Gaffé, J., Hallin, P.F., Riley, M.A., Lenski, R.E., and Schneider, D. (2010). Parallel genetic and phenotypic evolution of DNA superhelicity in experimental populations of Escherichia coli. Mol. Biol. Evol. 27, 2113-2128.

Cvijović, I., Nguyen Ba, A.N., and Desai, M.M. (2018). Experimental studies of evolutionary dynamics in microbes. Trends Genet. 34, 693-703.

Deatherage, D.E., and Barrick, J.E. (2014). Identification of mutations in laboratory-evolved microbes from next-generation sequencing data using breseq. Methods Mol. Biol. 1151, 165188.

Deatherage, D.E., Traverse, C.C., Wolf, L.N., and Barrick, J.E. (2015). Detecting rare structural variation in evolving microbial populations from new sequence junctions using breseq. Front. Genet. 5, 468.

Deatherage, D.E., Kepner, J.L., Bennett, A.F., Lenski, R.E., and Barrick, J.E. (2017). Specificity 
of genome evolution in experimental populations of Escherichia coli evolved at different temperatures. Proc. Natl. Acad. Sci. U. S. A. 114, E1904-E1912.

Desai, M.M., Walczak, A.M., and Fisher, D.S. (2012). Genetic diversity and the structure of genealogies in rapidly adapting populations. Genetics 193, 565-585.

Dinardo, S., Voelkel, K.A., Sternglanz, R., Reynolds, A.E., and Wright, A. (1982). Escherichia coli DNA topoisomerase I mutants have compensatory mutations in DNA gyrase genes. Cell 31, 43-51.

Ding, L., Ley, T.J., Larson, D.E., Miller, C. a., Koboldt, D.C., Welch, J.S., Ritchey, J.K., Young, M. a., Lamprecht, T., McLellan, M.D., et al. (2012). Clonal evolution in relapsed acute myeloid leukaemia revealed by whole-genome sequencing. Nature 481, 506-510.

Donovan, K.A., Atkinson, S.C., Kessans, S.A., Peng, F., Cooper, T.F., Griffin, M.D.W., Jameson, G.B., and Dobson, R.C.J. (2016). Grappling with anisotropic data, pseudo-merohedral twinning and pseudo-translational noncrystallographic symmetry: A case study involving pyruvate kinase. Acta Crystallogr. Sect. D Struct. Biol. 72, 512-519.

Fischer, S., Greipel, L., Klockgether, J., Dorda, M., Wiehlmann, L., Cramer, N., and Tümmler, B. (2017). Multilocus amplicon sequencing of Pseudomonas aeruginosa cystic fibrosis airways isolates collected prior to and after early antipseudomonal chemotherapy. J. Cyst. Fibros. 16, $346-352$.

Fowler, D.M., and Fields, S. (2014). Deep mutational scanning: a new style of protein science. Nat. Methods 11, 801-807.

Frenkel, E.M., McDonald, M.J., Van Dyken, J.D., Kosheleva, K., Lang, G.I., and Desai, M.M. (2015). Crowded growth leads to the spontaneous evolution of semistable coexistence in laboratory yeast populations. Proc. Natl. Acad. Sci. 112, 11306-11311.

Furusawa, C., Horinouchi, T., and Maeda, T. (2018). Toward prediction and control of antibiotic-resistance evolution. Curr. Opin. Biotechnol. 54, 45-49.

Garst, A.D., Bassalo, M.C., Pines, G., Lynch, S.A., Halweg-Edwards, A.L., Liu, R., Liang, L., Wang, Z., Zeitoun, R., Alexander, W.G., et al. (2017). Genome-wide mapping of mutations at single-nucleotide resolution for protein, metabolic and genome engineering. Nat. Biotechnol. 35, $48-55$.

Genovese, G., Kähler, A.K., Handsaker, R.E., Lindberg, J., Rose, S.A., Bakhoum, S.F., Chambert, K., Mick, E., Neale, B.M., Fromer, M., et al. (2014). Clonal hematopoiesis and bloodcancer risk inferred from blood DNA sequence. N. Engl. J. Med. 371, 2477-2487.

Gerrish, P.J., and Lenski, R.E. (1998). The fate of competing beneficial mutations in an asexual population. Genetica $102-103,127-144$.

Good, B.H., McDonald, M.J., Barrick, J.E., Lenski, R.E., and Desai, M.M. (2017). The dynamics of molecular evolution over 60,000 generations. Nature 551, 45-50.

Gresham, D., and Dunham, M.J. (2014). The enduring utility of continuous culturing in experimental evolution. Genomics 104, 399-405.

Hegreness, M., and Kishony, R. (2007). Analysis of genetic systems using experimental 
814 evolution and whole-genome sequencing. Genome Biol. 8, 201.

815 Hegreness, M., Shoresh, N., Hartl, D., and Kishony, R. (2006). An equivalence principle for the

816 incorporation of favorable mutations in asexual populations. Science 311, 1615-1617.

817 Hong, J., Brandt, N., Abdul-Rahman, F., Yang, A., Hughes, T., and Gresham, D. (2018). An

818 incoherent feedforward loop facilitates adaptive tuning of gene expression. Elife 7, 1-18.

819 Houdaigui, B. El, Forquet, R., Hindré, T., Schneider, D., Nasser, W., Reverchon, S., and Meyer,

820 S. (2019). Bacterial genome architecture shapes global transcriptional regulation by DNA

821 supercoiling. Nucleic Acids Res. 47, 5648-5657.

822 Jeong, H., Barbe, V., Lee, C.H., Vallenet, D., Yu, D.S., Choi, S.-H., Couloux, A., Lee, S.-W.,

823 Yoon, S.H., Cattolico, L., et al. (2009). Genome sequences of Escherichia coli B strains REL606

824 and BL21(DE3). J. Mol. Biol. 394, 644-652.

825 Khan, A.I., Dinh, D.M., Schneider, D., Lenski, R.E., and Cooper, T.F. (2011). Negative epistasis

826 between beneficial mutations in an evolving bacterial population. Science 332, 1193-1196.

827 Kochanowski, K., Volkmer, B., Gerosa, L., Van Rijsewijk, B.R.H., Schmidt, A., and

828 Heinemann, M. (2013). Functioning of a metabolic flux sensor in Escherichia coli. Proc. Natl.

829 Acad. Sci. U. S. A. 110, 1130-1135.

830 Kryazhimskiy, S., Rice, D.P., Jerison, E.R., and Desai, M.M. (2014). Global epistasis makes

831 adaptation predictable despite sequence-level stochasticity. Science 344, 1519-1522.

832 Kurnasov, O. V., Polanuyer, B.M., Ananta, S., Sloutsky, R., Tam, A., Gerdes, S.Y., and

833 Osterman, A.L. (2003). Ribosylnicotinamide Kinase Domain of NadR Protein: Identification and 834 Implications in NAD Biosynthesis. J. Bacteriol. 185, 698-698.

835 Landau, D.A., Carter, S.L., Stojanov, P., McKenna, A., Stevenson, K., Lawrence, M.S., 836 Sougnez, C., Stewart, C., Sivachenko, A., Wang, L., et al. (2013). Evolution and impact of 837 subclonal mutations in chronic lymphocytic leukemia. Cell 152, 714-726.

838 Lang, G.I., Rice, D.P., Hickman, M.J., Sodergren, E., Weinstock, G.M., Botstein, D., and Desai, 839 M.M. (2013). Pervasive genetic hitchhiking and clonal interference in forty evolving yeast 840 populations. Nature 500, 571-574.

841 Lee, S.Y., and Kim, H.U. (2015). Systems strategies for developing industrial microbial strains. 842 Nat. Biotechnol. 33, 1061-1072.

843 Lenski, R.E., and Travisano, M. (1994). Dynamics of adaptation and diversification: a 10,000evolution in Escherichia coli. I. Adaptation and divergence during 2,000 generations. Am. Nat. $138,1315-1341$. 
Marusyk, A., Almendro, V., and Polyak, K. (2012). Intra-tumour heterogeneity: A looking glass for cancer? Nat. Rev. Cancer 12, 323-334.

855

856

857

858

859

860

861

862

863

864

865

866

867

868

869

870

871

872

873

874

875

876

877

878

879

880

881

882

883

884

885

886

887

888

889

890

891

Marvig, R.L., Sommer, L.M., Molin, S., and Johansen, H.K. (2015). Convergent evolution and adaptation of Pseudomonas aeruginosa within patients with cystic fibrosis. Nat. Genet. 47, 5764.

Massé, E., and Drolet, M. (1999). Relaxation of transcription-induced negative supercoiling is an essential function of Escherichia coli DNA topoisomerase I. J. Biol. Chem. 274, 16654-16658.

Mattevi, A., Valentini, G., Rizzi, M., Speranza, M.L., Bolognesi, M., and Coda, A. (1995).

Crystal structure of Escherichia coli pyruvate kinase type I: molecular basis of the allosteric transition. Structure 3, 729-741.

McDonald, M.J. (2019). Microbial experimental evolution - a proving ground for evolutionary theory and a tool for discovery. EMBO Rep. 20, 1-14.

Merlo, L.M.F., Pepper, J.W., Reid, B.J., and Maley, C.C. (2006). Cancer as an evolutionary and ecological process. Nat. Rev. Cancer 6, 924-935.

Merlo, L.M.F., Shah, N.A., Li, X., Blount, P.L., Vaughan, T.L., Reid, B.J., and Maley, C.C. (2010). A comprehensive survey of clonal diversity measures in Barrett's esophagus as biomarkers of progression to esophageal adenocarcinoma. Cancer Prev. Res. (Phila). 3, 13881397.

Newman, A.M., Bratman, S. V., To, J., Wynne, J.F., Eclov, N.C.W., Modlin, L.A., Liu, C.L., Neal, J.W., Wakelee, H.A., Merritt, R.E., et al. (2014). An ultrasensitive method for quantitating circulating tumor DNA with broad patient coverage. Nat. Med. 20, 548-554.

Newman, A.M., Lovejoy, A.F., Klass, D.M., Kurtz, D.M., Chabon, J.J., Scherer, F., Stehr, H., Liu, C.L., Bratman, S. V., Say, C., et al. (2016). Integrated digital error suppression for improved detection of circulating tumor DNA. Nat. Biotechnol. 34, 547-555.

Nielsen, J., and Keasling, J.D. (2016). Engineering cellular metabolism. Cell 164, 1185-1197.

Ostrowski, E.A., Woods, R.J., and Lenski, R.E. (2008). The genetic basis of parallel and divergent phenotypic responses in evolving populations of Escherichia coli. Proc. R. Soc. B 275, $277-284$.

Park, S.-C., and Krug, J. (2007). Clonal interference in large populations. Proc. Natl. Acad. Sci. U. S. A. 104, 18135-18140.

Peng, F., Widmann, S., Wünsche, A., Duan, K., Donovan, K.A., Dobson, R.C.J., Lenski, R.E., and Cooper, T.F. (2018). Effects of beneficial mutations in $p y k F$ gene vary over time and across replicate populations in a long-term experiment with bacteria. Mol. Biol. Evol. 35, 202-210.

Perry, K., and Mondragón, A. (2003). Structure of a complex between E. coli DNA topoisomerase I and single-stranded DNA. Structure 11, 1349-1358.

Pruss, G.J., Manes, S.H., and Drlica, K. (1982). Escherichia coli DNA topoisomerase I mutants: Increased supercoiling is corrected by mutations near gyrase genes. Cell 31, 35-42.

R Core Team (2016). R: A Language and Environment for Statistical Computing (Vienna, Austria: R Foundation for Statistical Computing). 
Raeside, C., Gaffé, J., Deatherage, D.E., Tenaillon, O., Briska, A.M., Ptashkin, R.N., Cruveiller, S., Médigue, C., Lenski, R.E., Barrick, J.E., et al. (2014). Large chromosomal rearrangements during a long-term evolution experiment with Escherichia coli. MBio 5, e01377-14. (1999). The Escherichia coli NadR regulator is endowed with nicotinamide mononucleotide adenylyltransferase activity. J. Bacteriol. 181, 5509-5511. experimental evolution? Curr. Opin. Genet. Dev. 47, 102-109.

900 Renda, B.A., Hammerling, M.J., and Barrick, J.E. (2014). Engineering reduced evolutionary 901 potential for synthetic biology. Mol. Biosyst. 10, 1668-1678.

902 Rugbjerg, P., and Sommer, M.O.A. (2019). Overcoming genetic heterogeneity in industrial fermentations. Nat. Biotechnol. 37, 869-876.

904 Rugbjerg, P., Myling-Petersen, N., Porse, A., Sarup-Lytzen, K., and Sommer, M.O.A. (2018). 905 Diverse genetic error modes constrain large-scale bio-based production. Nat. Commun. 9, 787.

906 Ryall, B., Eydallin, G., and Ferenci, T. (2012). Culture history and population heterogeneity as determinants of bacterial adaptation: the adaptomics of a single environmental transition. Microbiol. Mol. Biol. Rev. 76, 597-625.

Sandoval, C.M., Ayson, M., Moss, N., Lieu, B., Jackson, P., Gaucher, S.P., Horning, T., Dahl, increases the genetic stability of farnesene producing Saccharomyces cerevisiae. Metab. Eng. 25, 912 215-226.

913 Schmitt, M.W., Kennedy, S.R., Salk, J.J., Fox, E.J., Hiatt, J.B., and Loeb, L.A. (2012). Detection

914 of ultra-rare mutations by next-generation sequencing. Proc. Natl. Acad. Sci. U. S. A. 109, $91514508-14513$.

916 Siddiquee, K.A.Z., Arauzo-Bravo, M.J., and Shimizu, K. (2004). Effect of a pyruvate kinase ( $p y k F$-gene) knockout mutation on the control of gene expression and metabolic fluxes in

918 Escherichia coli. FEMS Microbiol. Lett. 235, 25-33.

919 Stefani, S., Campana, S., Cariani, L., Carnovale, V., Colombo, C., Lleo, M.M., Iula, V.D., 920 Minicucci, L., Morelli, P., Pizzamiglio, G., et al. (2017). Relevance of multidrug-resistant 921 Pseudomonas aeruginosa infections in cystic fibrosis. Int. J. Med. Microbiol. 307, 353-362.

922 Tan, K., Zhou, Q., Cheng, B., Zhang, Z., Joachimiak, A., and Tse-Dinh, Y.C. (2015). Structural 923 basis for suppression of hypernegative DNA supercoiling by E. coli topoisomerase I. Nucleic 924 Acids Res. 43, 11031-11046.

925 Tenaillon, O., Rodríguez-Verdugo, A., Gaut, R.L., McDonald, P., Bennett, A.F., Long, A.D., and 926 Gaut, B.S. (2012). The molecular diversity of adaptive convergence. Science 335, 457-461.

927 Tenaillon, O., Barrick, J.E., Ribeck, N., Deatherage, D.E., Blanchard, J.L., Dasgupta, A., Wu, 928 G.C., Wielgoss, S., Cruveiller, S., Médigue, C., et al. (2016). Tempo and mode of genome 929 evolution in a 50,000-generation experiment. Nature 536, 165-170.

930 Thomas, R.K., Nickerson, E., Simons, J.F., Jänne, P.A., Tengs, T., Yuza, Y., Garraway, L.A., 
931 LaFramboise, T., Lee, J.C., Shah, K., et al. (2006). Sensitive mutation detection in heterogeneous 932 cancer specimens by massively parallel picoliter reactor sequencing. Nat. Med. 12, 852-855.

933 Traverse, C.C., Mayo-Smith, L.M., Poltak, S.R., and Cooper, V.S. (2013). Tangled bank of 934 experimentally evolved Burkholderia biofilms reflects selection during chronic infections. Proc.

935 Natl. Acad. Sci. U. S. A. 110, E250-E259.

936 Venkataram, S., Dunn, B., Li, Y., Agarwala, A., Chang, J., Ebel, E.R., Geiler-Samerotte, K., 937 Hérissant, L., Blundell, J.R., Levy, S.F., et al. (2016). Development of a comprehensive 938 genotype-to-fitness map of adaptation-driving mutations in yeast. Cell 166, 1585-1596.E22.

939 Watson, C.J., Papula, A.L., Poon, G.Y.P., Wong, W.H., Young, A.L., Druley, T.E., Fisher, D.S., 940 and Blundell, J.R. (2020). The evolutionary dynamics and fitness landscape of clonal

941 hematopoiesis. Science 367, 1449-1454.

942 Wei, X., and Zhang, J. (2019). Patterns and mechanisms of diminishing returns from beneficial 943 mutations. Mol. Biol. Evol. 36, 1008-1021.

944 Wickham, H. (2016). ggplot2: Elegant Graphics for Data Analysis (New York: Springer-Verlag).

945 Winstanley, C., O’Brien, S., and Brockhurst, M.A. (2016). Pseudomonas aeruginosa

946 evolutionary adaptation and diversification in cystic fibrosis chronic lung infections. Trends

947 Microbiol. 24, 327-337.

948 Wiser, M.J., Ribeck, N., and Lenski, R.E. (2013). Long-term dynamics of adaptation in asexual 949 populations. Science 342, 1364-1367.

950 Woods, R., Schneider, D., Winkworth, C.L., Riley, M.A., and Lenski, R.E. (2006). Tests of 951 parallel molecular evolution in a long-term experiment with Escherichia coli. Proc. Natl. Acad. 952 Sci. U. S. A. 103, 9107-9712.

953 Woods, R.J., Barrick, J.E., Cooper, T.F., Shrestha, U., Kauth, M.R., and Lenski, R.E. (2011). 954 Second-order selection for evolvability in a large Escherichia coli population. Science 331, 955 1433-1436.

956 Zelder, O., and Hauer, B. (2000). Environmentally directed mutations and their impact on 957 industrial biotransformation and fermentation processes. Curr. Opin. Microbiol. 3, 248-251. 